EPJ manuscript No.

(will be inserted by the editor)

\title{
A Machian Solution of the Hierarchy Problem
}

\author{
Merab Gogberashvili \\ 1 Andronikashvili Institute of Physics, 6 Tamarashvili St., Tbilisi 0177, Georgia \\ 2 Javakhishvili State University, 3 Chavchavadze Avenue, Tbilisi 0128, Georgia
}

Received:

\begin{abstract}
The new interpretation of Mach's principle of mass of a particle being a measure of the interactions of this particle with all other gravitating particles inside its causal spheres is introduced. It is shown that within some alternative model of gravitation that incorporates this principle, the Machian influence of the universe can reduce Planck's scale to the electro-weak scale and the large number that is needed to explain the hierarchy between the scales is the amount of gravitating particles inside the universe horizon. Our model can lead to new observable effects at cosmological distances and close to the sources of a strong gravitational field.
\end{abstract}

PACS. $04.20 . \mathrm{Cv}$ 04.90.+e 98.80.-k

In particle physics the hierarchy problem mainly consists in the existence of a large separation between the electro-weak and Planck's scales, or equivalently between the Higgs and Planck masses. The Higgs mass is quadratically sensitive to the cut-off of the theory and if the cut-off is very high it is not clear what stabilizes it. If the cut-off is not far from the electro-weak scale, then one has to explain why gravity is so weak. In both cases, the solution should come up in the form of the large number $N$ that provides the hierarchy between the Planck mass $M_{P}$ and the Higgs mass $M_{H}$,

$$
M_{P}^{2} \approx N M_{H}^{2}
$$

For example, in SUSY the large number $N$ is the ratio of Planck's mass to the supersymmetry breaking scale, whereas in large extra dimensions scenarios the large volume of the extra space sets the hierarchy.

In the recent papers [1,2] there was considered a different approach, in which $N$ in (1) is the number of species of some new quantum fields. The argument of $[1$ is that each $N$ species of fields, with masses at the scale $M_{H}$, coupled to gravity will contribute to the renormalization of Planck's mass the factor $\sim M_{H}^{2}$. Neglecting the accidental cancellations this has to be multiplied by the number of species. As a result the effective contribution to the Planck mass is $\sim N M_{H}^{2}$. Later in [2] also a non-perturbative argument was introduced: in the model with a large amount of different quantum fields Planck's mass violating the bound (11) is inconsistent with black hole physics.

Here we want to elaborate another idea of how to reduce Planck's scale to the electro-weak scale. The model based on Mach's effect asserting that any local gravitation interaction is affected by all matter in the universe [3]. We shall show that Mach's effect can reduce the local strength of the gravitational interaction, and the large number $N$ in (11) can be the amount of gravitating particles within the universe horizon. Note that up to astrophysical distances this model does not requires radical changes of standard physics, such as the introduction of extra dimensions, or new interactions.

Mach's principle was considered by Einstein in the development of general relativity [4 but was rejected when he found that his field equations admit curved vacuum and asymptotically flat solutions. Mach's principle deals with a fundamental issue of physics: the origin of inertia (or the nature of inertial mass). General relativity does not explain the origins of inertial and gravitational masses; it just states that they are equivalent. According to Mach some close mass, like a galaxy, should lead to anisotropy of the inertial mass $m_{i}$ of a body. However, precise tests do not show this difference; for example, it was found that $\Delta m_{i} / m_{i} \leq 10^{-20}$ for nucleons [5]. So the existing data are in favor of Einstein's equivalence principle and against a classical interpretation of Mach's effect. Discussions on the present status of Mach's principle can be found in [6.

In our opinion contradictions with Einstein's equivalence principle can be resolved if we use Mach's ideas on the level of particle physics assuming strong non-locality in a future quantum theory of gravity. We suppose that the mass (inertial and gravitational) of any particle is a measure of the interactions of this particle with all other gravitating particles inside its causal sphere and not with some classical massive objects like distant stars. Since for the distances of the order of the horizon the distribution of matter is isotropic (cosmological principle), local fluctuations of the gravitational potential (or the existence of close masses) does not affect the description of the inertial mass of a particle. 
In particle physics, mass is a measure of the coupling of particles with the Higgs field and it is usually assumed that all particles in the universe receive there masses by the same mechanism. It is natural to suppose that both the inertial and gravitational masses are connected with the fundamental Higgs scale, and Newton's constant is some emergent quantity. So in the model that incorporates Mach's principle in a novel interpretation the universal background Higgs field probably can be replaced by the background gravitational potential of the universe.

The main observation is that the inertial $m_{i}$ and the gravitational $m_{g}$ masses in general are not necessarily equivalent. One can state only that these masses of an object are proportional to one another:

$$
m_{g}=\sqrt{N} m_{i},
$$

where $N$ is some constant of proportionality. This is usually referred as the 'weak form' of the equivalence principle. The assumption (2) does not means a violation of the equivalence principle since $N$ is still the same for all bodies, whatever their compositions are. In Einstein's theory it is assumed that inertial and gravitational masses of any object are exactly equivalent, i.e. $N=1$. However, all the results of standard physics would still remain valid if we write (2) and at the same time introduce the new gravitational constant

$$
g=N G,
$$

where $G$ is usual Newton's constant.

Equation (3) is a relation similar to (11). We assume that, because of Machian effects, the constant $g$ in (3) can be connected with the electro-weak scale, i. e.

$$
g \sim \frac{1}{M_{H}^{2}},
$$

and the large quantity $N$ is the number of gravitating particles within the horizon that are sources of gravitational interaction. If this mechanism works it can explain some large number coincidence in physics. A recent review on the large numbers hypothesis, proving the existence of some deep connection between micro and macro physics, the reader can find in [7.

Another observation is that mass is a positive quantity and screening of gravity is impossible. The 'gravitational flux' of a body should be conserved and this flux will distribute to all interacting particles. So in any twobody process the influence of all other matter inside their causal sphere should be taken into account. Because of the existence of a large amount of matter in the universe this should effectively reduce the strength of any two-body interaction, i.e. the gravitational constant.

When considering the gravitational interaction of close objects one can replace the distant universe by a spherical shell of the effective mass $M$ and the effective radius $R$. This physical substitution is analogous to replacing of a spherical mass by a point mass. As in the electrical case this shell acts similar to a gravitational Faraday cage inside of which a huge, yet constant, gravitational potential exists,

$$
\phi=-\frac{M G}{R} .
$$

Since the potential (5) is constant the 'universe field' $E$ on an inertial particle is zero,

$$
\nabla \phi=0 .
$$

However, accelerated particles feel the constant 'universe potential' (5) and similar to the induction law in electrodynamics for the field strength we have 8

$$
E=-\nabla \phi-\frac{\phi}{c^{2}} a=-\frac{\phi}{c^{2}} a,
$$

where $a$ is the vector of acceleration and $c$ is the speed of light.

In the case of a homogeneous and isotropic matter distribution in the universe with the average density $\rho$ we have the following relationships:

$$
\frac{\phi}{c^{2}}=-\frac{G \rho V}{R c^{2}}=-\frac{2 \pi G \rho R^{2}}{c^{2}}=-\frac{2 \pi G \rho}{H^{2}} .
$$

For simplicity the considered volume of the universe is a Hubble sphere of radius

$$
R=\frac{c}{H} .
$$

Using the formula for the critical mass density of the universe

$$
\rho_{c}=\frac{3 H^{2}}{8 \pi G}
$$

from (8) we found that the relationship

$$
\phi \approx-c^{2}
$$

is valid to a reasonable degree of precision for our simple considerations. It seems that the relation (11) is valid for all stages of the universe expansion and the gravitational potential in the universe remains unchanged (conserved) since the Planck time [9],

$$
\phi=-\frac{G M_{P}}{l_{P}}=-c^{2},
$$

where $l_{P}$ is Planck's length. This formula in fact coincides with the definition of Newton's constant by Planck's mass

$$
G \sim \frac{1}{M_{P}^{2}}
$$

in units where $c=1$.

The gravitational field of the universe (7) on a particle with the mass $m_{g}$ due to (11) results in the standard expression of the inertial force,

$$
F=m_{g} E=m_{i} a,
$$

where the notion of inertial mass, as the measure of the gravitational interaction with the universe, was introduced by

$$
m_{i}=-m_{g} \frac{\phi}{c^{2}} .
$$


This formula provides support to Mach's hypothesis about the origin of inertia.

One can also obtain (11) from Friedman's equations by the integration of the deceleration vector along the radius of the causal sphere from the center to the particle horizon [10. The relation (11) can be understood as the definition of the horizon of the universe - the internal horizon of the Schwarzschild sphere, or alternatively as the distance at which the velocity of recession of galaxies is equal to the speed of light.

The relation (11) leads also to another conclusion that the total energy (inertial plus gravitational) of a particle at rest to the universe is zero [8],

$$
m_{i} c^{2}+m_{g} \phi=0 \text {. }
$$

One can show that this relation is equivalent to the standard definition of inertial coordinate system as the frame in which all forces on a body compensate.

The fact of conservation of the potential $\phi$ during expansion raises the question of the variation of the gravitational constant $G$, since only in a very specific cosmological model the mass of the universe $M$ increases linearly with the radius $R$. While the macroscopic characteristics of the universe can vary during the expansion, it is usually assumed that the parameters of particle physics are unaffected. So if $g$ in (3) is connected with the electro-weak scale it should remain constant, but the number $N$ and Newton's constant $G$ can change in time. The possibility of a variation of $G$ was considered by many authors (for a review see [11).

To estimate $N$, and thus $g$, let us consider the spherical model universe of radius $R$ consisting of $N$ uniformly distributed particles of gravitational mass $m_{g}$. Each particle in our toy model gravitationally 'feels' all the other $(N-1)$ particles. The mean contribution of each pair to the gravitational energy will be $m_{g}^{2} / R$. Thus the 'universe potential' will contain $\approx N^{2}$ terms formed by all pairs of particles, for which the mean separation will be $R$, i. e.

$$
\phi \approx-\frac{m_{g} G}{R} N^{2} .
$$

Comparing this formula with (5), we see that due to the interactions the active gravitational mass of the universe in this model is equal to

$$
M=N^{2} m_{g}
$$

and not to

$$
\int \rho d V=N m_{g}
$$

as expected from Gauss's law in the additive case.

From the relation (17) we see that for small values of $N$, if we state that $m_{i}=m_{g}$ and $M=N m_{g}$, gravity gets stronger by the factor $N$. Thus the gravity scale increases with decreasing of $N$ according to the law (3) and achieves its maximal value $g$ in the case of the presence of only two particles in the universe. This means that the real interaction scale in this model is $g \sim 1 / M_{H}^{2}$ and $G$ is some emergent quantity incorporating the influence of all particles on any local interaction.

To estimate the number $N$ we need to calculate the amount of gravitating particles (including dark components) within the Hubble horizon. The inertial mass of a typical particle, for example a proton, has the order $m_{p} \sim 10^{-27} \mathrm{~kg}$. From the estimations of the critical mass density (10), it is known that $M \sim 10^{53} \mathrm{~kg}$. From (18) it follows that the gravitational mass of the universe $M$ is $N$ times larger than the sum of the masses of all particles. So the proton equivalent of the total number of gravitating particles is

$$
N \sim \sqrt{\frac{M}{m_{p}}} \sim 10^{40} .
$$

This is a large number of the order that one needs to reduce Planck's scale to the electro-weak scale by the formula (3).

Another toy model that shows that $g$ in (3) really can be connected with the electro-weak scale is the universe filled with $N$ particles of the masses $m_{g}$ half of which carry positive unit charges $e$ and the other half negative charges $-e(N$ is an even integer) [12. It is known that the intensity of electromagnetic interaction of two elementary particles is $10^{40}$ times larger than their gravitational interaction for the same distance,

$$
\frac{k e^{2}}{G m_{g}^{2}} \sim 10^{40}
$$

where $k$ is Coulomb's constant. However, to compare the total intensities one should take into account the gravitational interaction of all particles in the universe. In estimations of the potentials the fact that all masses are positive, while half the charges are positive and half negative leads to large differences. The gravitational potential expressed by (17) contains $\approx N^{2}$ terms. In contrast, each charge $e$ beyond the Debye radius finds itself in a neutral cloud. In fact, the total charge outside any considered charge $e$ will be $-e$, and the 'mean distance' of the opposite charge will be $R$. Therefore, the electrostatic potential consists of $N$ terms, each of magnitude $\approx e^{2} / R$. So the fundamental gravitational interaction engages all $N$ particles and only two electromagnetic ones. Thus the ratio of the total gravitational and electromagnetic intensities is close to unity, i. e.

$$
\frac{k e^{2}}{g m_{g}^{2}} \sim 1 \text {. }
$$

From this estimation we can conclude that in this model the Coulomb constant $k$ and the fundamental gravitational constant $g$ are connected to the same electro-weak scale.

Discussions: In this paper the new interpretation of Mach's principle that the mass of a particle is a measure of the interactions of this particle with all other gravitating particles inside its causal sphere was introduced. It was shown that within some alternative theory of gravitation, which assumes this non-local Machian response of the universe in local experiments, one can explain the observed 
hierarchy between the scales of particle physics and gravity. The reason why gravity seems to be weak in two-body interactions is that the mass (the charge of gravity) is always positive. Then screening of gravity is impossible and the gravitational flux of any body is distributed over all matter inside the horizon. It was found that in this model the large number needed for effective weakening of the gravitational constant from the electro-weak to Planck's scale coincides with the amount of gravitating particles in the universe. Note that, unlike other models with a single scale for particle physics and gravity, the incorporation of Mach's principle does not requires radical changes of standard physics up to astrophysical distances.

Since we want to reduce the fundamental scale of gravity, the model presented here can lead to observable new effects in high energy physics experiments, or for strong gravitational fields. In the cosmological case the modification of the description of mass can imitate the effects of dark energy at ultra-large distances.

I would like to thank Prof. Z. Berezhiani for helpful discussions.

\section{References}

1. G. Dvali, G. Gabadadze, M. Kolanović and F. Nitti, Phys. Rev. D 65, (2002) 024031 [arXiv: hep-th/0106058].

2. G. Dvali, arXiv: 0706.2050 [hep-th].

3. E. Mach, The Science of Mechanics - a Critical and Historical Account of its Development (Open Court, La Salle 1960).

4. A. Einstein, The meaning of relativity, 6th edition (Chapman \& Hall, London 1991).

5. V. W. Hughes, H. G. Robinson and V. Beltran-Lopez, Phys. Rev. Lett. 4, (1960) 342;

R. W. P. Drever, Phil. Mag. 6, (1961) 683.

6. J. Barbour and H. Pfister, Mach's Principle: From Newton's Bucket to Quantum Gravity (Birkhäuser, Boston 1995) Einstein studies, vol. 6;

A. Ghosh, Origin of Inertia (Apeiron, Montreal 2000).

7. S. Ray, U. Mukhopadhyay and P. P. Ghosh, arXiv: 0705.1836 [gr-qc].

8. D. W. Sciama, Mon. Not. R. Astron. Soc. 113, (1953) 34; Sci. Amer. 196, (1957) 99; The Physical Foundations of General Relativity (Doubleday, New York 1969); Modern Cosmology (Cambridge Univ. Press, London 1971).

9. I. R. Kenyon, General Relativity (Oxford Univ. Press, Oxford 1990).

10. R. L. Signore, Nuovo Cimento 111, (1996) 1087; Nuovo Cimento 112, (1996) 1593.

11. J.-P. Uzan, Rev. Mod. Phys. 75, (2003) 403 [arXiv: hep-ph/0205340.

12. J. Barbour, Class. Quant. Grav. 20, (2003) 1543 [arXiv: gr-qc/0211021. 